\title{
Rasmussen's aneurysm: a rare and potentially fatal cause of hemoptysis
}

\begin{abstract}
Rasmussen's aneurysm is a rare and fatal cause of hemoptysis secondary to infection with pulmonary tuberculosis. The most commonly involved vessels include the bronchial arteries, but rarely can involve the pulmonary artery.

We report the case of a 62-year-old female from the Philippines with undiagnosed pulmonary tuberculosis who presented with massive hemoptysis. After hemodynamic stabilization, Rasmussen's aneurysm was diagnosed by computed tomography of the chest with angiography, confirmed with invasive angiography. She was treated definitively with glue embolization of the affected artery.
\end{abstract}

Key words: hemoptysis, tuberculosis, critical care, angiography

Adv Respir Med. 2021; 89: 581-584

\section{Introduction}

Tuberculosis (TB), caused by the bacterium Mycoplasma tuberculosis, is an infectious disease with significant global burden, with approximately $25 \%$ of the world's population estimated to be infected [1]. Multidrug resistant strains have become an increasing problem complicating treatment. In the United States, however, the incidence rate of TB has been consistently declining for at least the past fifty years. In 2019, the United States' TB rate declined to its lowest rate yet, at 2.7 cases per 100,000 persons [2]. A large portion of these cases are attributed to reactivation of latent TB infection. Because of its low incidence overall in the United States, and effective screening programs in high-risk populations, significant complications are rarely seen. We present a case of a patient with a previously undiagnosed TB infection who suffered massive hemoptysis due to invasive cavitation of the pulmonary parenchyma with involvement of an adjacent branch of the pulmonary artery, a phenomenon known as the Rasmussen's aneurysm.

\section{Case presentation}

A 62-year-old woman from the Philippines presented to the hospital with a complaint of dyspnea and hemoptysis. The patient initially began to have dyspnea at rest for several months. One month prior to presentation, she developed a productive cough. The patient was diagnosed by her primary care provider with "acute bronchitis" and was first given a course of levofloxacin, then three courses of amoxicillin. Despite this, she continued to have worsening cough with blood noted in the sputum, prompting her to come to the emergency department. Her prior medical history included hypertension and a history of breast cancer on tamoxifen therapy. She also had a history of multiple positive purified protein derivative (PPD) tests for TB in the past, but with no formal diagnosis or treatment of latent TB.

On initial physical examination, the patient was dyspneic but able to speak full sentences. Her oxygen saturation was $95 \%$ on room air. Pulmonary exam was remarkable for bilateral rhonchi to lung auscultation. The rest of physical exam was 
unremarkable. While the patient was waiting in the emergency department, she experienced an episode of massive hemoptysis, became hypoxic, and then bradycardic without pulse. Advanced cardiac life support was initiated, and she received four rounds of cardiopulmonary resuscitation with return of spontaneous circulation. Subsequently she was intubated, resuscitated with fluids, and then started on a norepinephrine drip for shock. Initial laboratory workup revealed a hemoglobin level of $8.8 \mathrm{~g} / \mathrm{dL}$ (baseline was $14 \mathrm{~g} / \mathrm{dL}$ ), white blood cell count of $19,600 / \mathrm{mm}^{3}$, and lactate of $8.8 \mathrm{mmol} / \mathrm{L}$. The patient was taken emergently for computed tomographic pulmonary angiography (CTPA) which showed an active pulmonary arterial bleed in the left lower lobe, with an associated $4.5 \mathrm{~cm}$ blood filled mass-like cavitary lesion in the left upper lobe in proximity with mentioned bleeding artery (Figures 1, 2). Invasive pulmonary angiography was performed confirming a large pseudoaneurysm arising from the proximal left lower lobe pulmonary artery with active bleeding into a cavitary lesion (Figure 3).

The patient underwent emergent glue embolization with coils placed in the remnant proximal left lower lobar pulmonary artery (Figure 4). Sputum and bronchoalveolar lavage (BAL) specimens were sent for acid-fast bacilli (AFB) direct and concentrated smears. These were positive for Mycobacterium tuberculosis complex without any drug resistance genes. The patient was started on a liver sparing anti-tuberculosis regimen as she showed evidence of acute hepatic injury, likely due to shock. The regimen included rifampin, ethambutol, and levofloxacin. She remained sedated and intubated for five days following the embolization. There were no further episodes of hemoptysis and her hemoglobin remained stable.

Unfortunately, the patient was diagnosed with a severe anoxic brain injury as a result of her initial cardiac arrest. Her family requested a change in her goals of care to comfort care. Her anti-tuberculosis regimen was maintained until her passing for infection control purposes.

\section{Discussion}

The differential diagnosis of life-threatening hemoptysis such as in this patient includes bronchiectasis (particularly in cystic fibrosis), fungal infections, tuberculosis, malignancy, and vascular disorders including the vasculitides [3, 4]. Based on our patient's previously positive PPD tests, imaging studies, and the fact that the patient lived in an endemic country for most of her life,

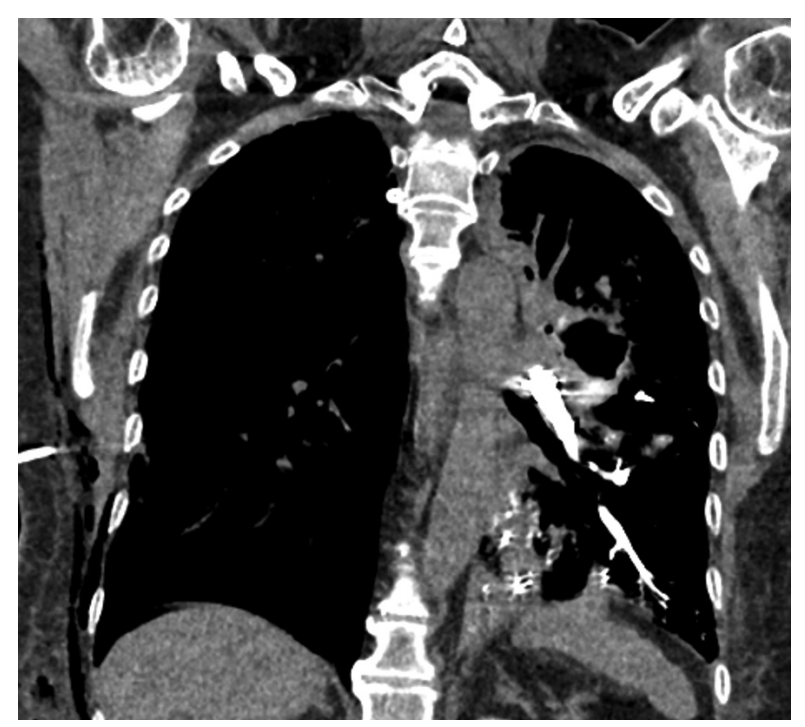

Figure 1. Coronal CT of the chest showing the mediastinal window of a left upper lobe cavitation in direct contact with the left lower branch of the pulmonary artery

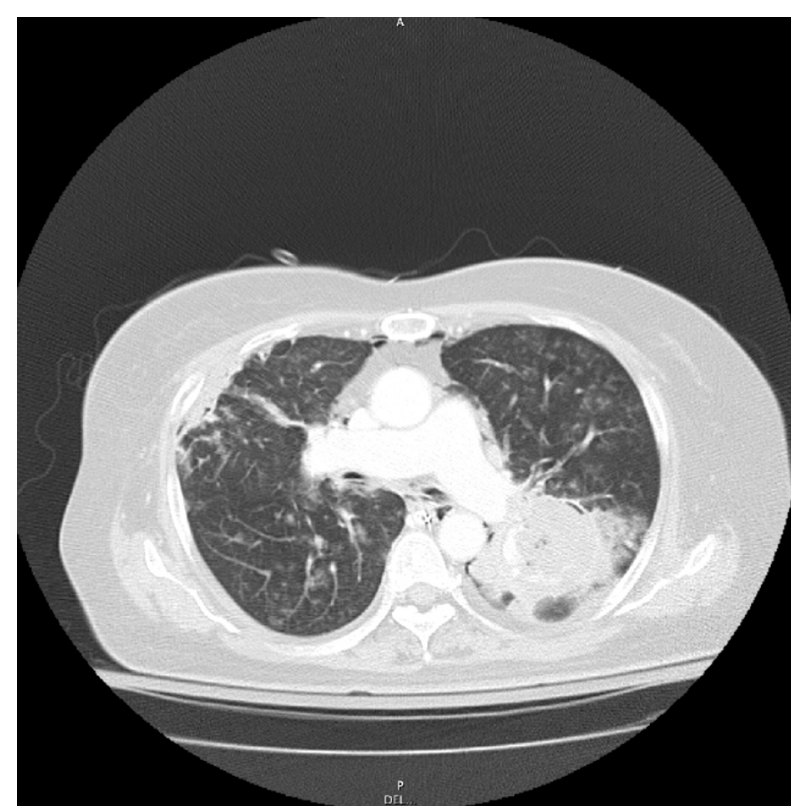

Figure 2. Transverse CT of the chest showing the lung window of a left upper lobe cavitation in direct contact with the left lower branch of the pulmonary artery

there was a high suspicion that the hemoptysis was secondary to TB infection. Pulmonary TB presents with a variety of symptoms, which are usually insidious in onset and progression. Symptoms include low-grade fever, night sweats, cough, weight loss and mild hemoptysis that usually persist for weeks before patients seek healthcare. Rarely, patients can present with massive hemoptysis, which has a mortality rate up to 


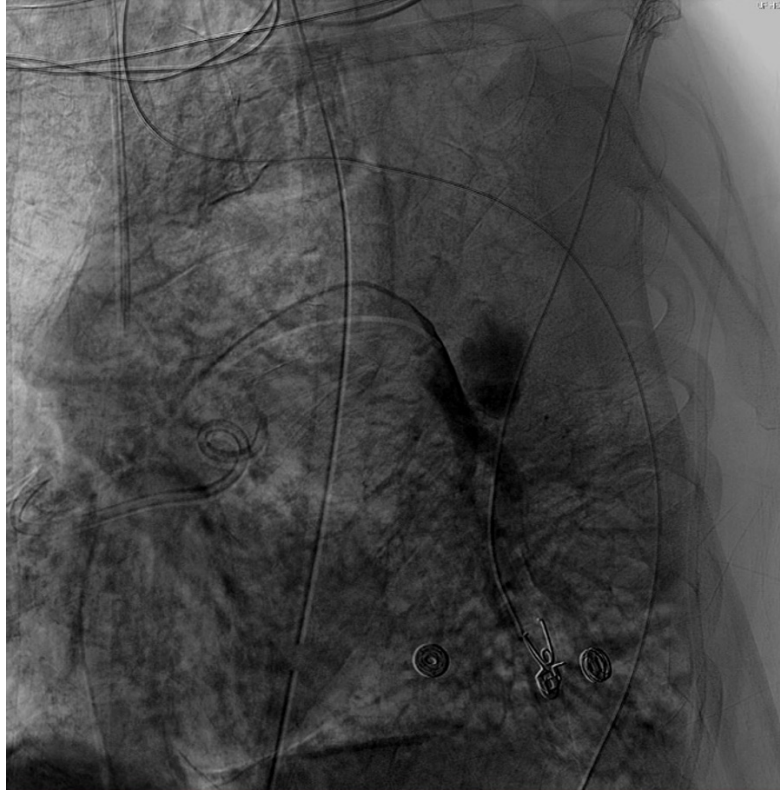

Figure 3. Angiography of the left upper lobe of the lung demonstrating active bleeding into the cavitary lesion

$50 \%$. Massive hemoptysis in tuberculosis can be caused by a phenomenon known as the Rasmussen's aneurysm, as was the case with our patient. Rasmussen's aneurysm is a potential complication of chronic cavitary pulmonary disease, due to the invasion into the pulmonary vasculature. In a post-mortem analysis of patients with chronic cavitary tuberculosis, it has been estimated that up to $4 \%$ of patients had pathology consistent with Rasmussen's aneurysm [4]. Of those with massive hemoptysis secondary to a Rasmussen's aneurysm, the most commonly involved vessels are the bronchial arteries. In less than $10 \%$ of cases, the main pulmonary artery may be involved.

Rasmussen's aneurysm is a pseudo-aneurysmal dilatation of a branch of pulmonary artery adjacent to a tuberculous cavity that can rupture and cause massive hemoptysis. As a tuberculous cavity begins to extend into the pulmonary artery, granulation tissue begins to invade and replace the adjacent pulmonary arterial wall (specifically the tunica adventitia and tunica media). Fibrin replacement of the vessel wall leads to thinning, and eventually herniates into the lumen of the vessel creating a pseudoaneurysm [5]. This pathology is reported to be associated with $5 \%$ of all tuberculous cavitary lesions [6]. The advent of contrast-enhanced CT has enabled a noninvasive, first-line method of localizing the site of arterial bleeding in the setting of massive hemoptysis, as seen in our case [7]. In our patient, both fiberoptic bronchoscopy and CTPA helped to identify the

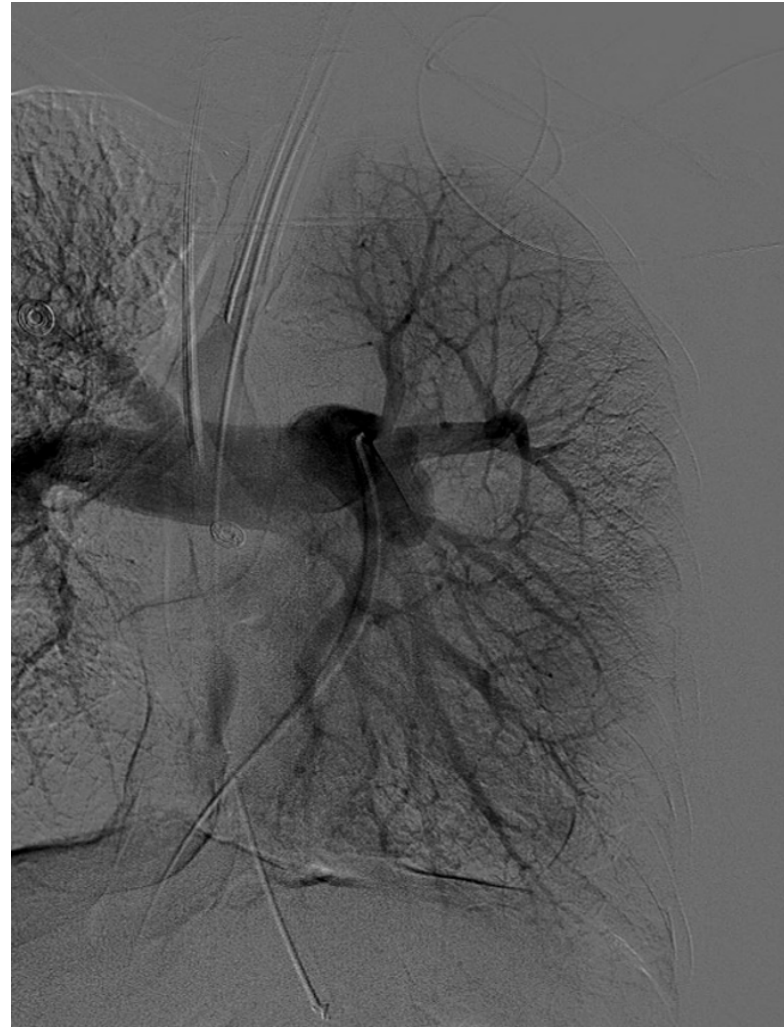

Figure 4. Post-embolization angiography of the left upper lobe of the lung demonstrating resolution of the bleeding pulmonary artery

site of bleeding from a pulmonary artery pseudoaneurysm, leading to prompt intervention.

After resuscitation and stabilization of the patient, endovascular management techniques like trans-arterial catheter embolization are the preferred management strategy. Embolization techniques can include glue embolization, coil packaging, gel foam, detachable balloons, or stent grafts [6]. In Rasmussen's aneurysm, glue embolization is the preferred technique due to the fragility of the artery [8].

\section{Conclusions}

Rasmussen's aneurysm is a rare complication of pulmonary TB that can cause life-threatening hemoptysis, particularly in non-endemic countries. In patients presenting with massive hemoptysis, it is important to include in the differential diagnosis, and treat rapidly with embolization. Glue embolization is the preferred trans-catheter technique due to the fragility of the involved vasculature.

\section{Conflict of interest}

None declared. 


\section{References:}

1. Houben RM, Dodd PJ. The global burden of latent tuberculosis infection: a re-estimation using mathematical modelling. PLoS Med. 2016; 13(10): e1002152, doi: 10.1371/journal. pmed.1002152, indexed in Pubmed: 27780211.

2. Schwartz NG, Price SF, Pratt RH, et al. Tuberculosis United States, 2019. MMWR Morb Mortal Wkly Rep. 2020; 69(11): 286-289, doi: 10.15585/mmwr.mm6911a3, indexed in Pubmed: 32191684.

3. Kim HY, Song KS, Goo JM, et al. Thoracic sequelae and complications of tuberculosis. Radiographics. 2001; 21(4): 839858, doi: 10.1148/radiographics.21.4.g01jl06839, indexed in Pubmed: 11452057.

4. Rajamannar KV, Kilaru H, Aravelly S, et al. Massive hemoptysis from Rasmussen's aneurysm in active pulmonary tuberculosis; A case report of successful treatment with bronchial artery embolization. Respir Med Case Rep. 2017; 22: 277-279, doi 10.1016/j.rmcr.2017.10.003, indexed in Pubmed: 29124005.

5. Sapra R, Sharma G, Minz AK. Rasmussen's aneurysm: A rare and forgotten cause of hemoptysis. Indian Heart J. 2015; 67 Suppl 3: S53-S56, doi: 10.1016/j.ihj.2015.07.009, indexed in Pubmed: 26995433.

6. Chatterjee A, Jackson AE, Acharya D, et al. Transcatheter closure of pulmonary arteriovenous malformation to facilitate treatment of pulmonary arterial hypertension. JACC Cardiovasc Interv. 2018; 11(6): e45-e46, doi: 10.1016/j. icin.2017.12.014, indexed in Pubmed: 29501545.

7. Keeling AN, Costello R, Lee MJ. Rasmussen's aneurysm: a forgotten entity? Cardiovasc Intervent Radiol. 2008; 31(1): 196-200, doi: 10.1007/s00270-007-9122-6, indexed in Pubmed: 17659424.

8. Garcia-Reina S, Martinez-Barenys C, Fernandez E. Giant Rasmussen's Aneurysm. Ann Thorac Surg. 2018; 106(4): e205, doi: 10.1016/.athoracsur.2018.03.032, indexed in Pubmed: 29673635. 